\title{
Extrarenal angiomyolipoma in an elderly male: A case to remember
}

Angiomyolipoma ( $A M L)$ is a benign mesenchymal tumor, mostly seen in kidneys and usually in association with tuberous sclerosis. Extrarenal AML is uncommon, but sporadic cases have been reported at other sites. Here, we are presenting a case of extrarenal AML in the right lower extremity of a 70-year-old male with no evidence of tuberous sclerosis.

Key words: Angiomyolipoma, extrarenal, lump, tuberous sclerosis

\section{INTRODUCTION}

Angiomyolipoma (AML) is a benign mesenchymal tumor composed of a variable proportion of adipose tissue, spindle and epithelioid smooth muscle cells (SMC) and abnormal thick-walled blood vessels. ${ }^{[1]}$ It is usually observed in the kidney of patients with tuberous sclerosis, a disease caused by loss-of-function mutations in the TSC1 or TSC2 tumor suppressor genes. ${ }^{[2]}$ Occurrence at other sites is uncommon and only few sporadic cases have been reported in liver, mediastinum, heart, spermatic cord, vaginal wall, fallopian tube, oral cavity, pharynx, nasal cavity, soft tissue, and skin. ${ }^{[2-4]}$ Extrarenal AML usually is not associated with tuberous sclerosis although it shares the terminology with renal AML. ${ }^{[5]}$ Here, we describe a case of AML that developed as a subcutaneous nodule in the right lower limb of an elderly male. The manuscript has been approved by Institutional Research and Ethics Committee.

\section{Brijesh Thakur, Sanjeev Kishore, Aparna Bhardwaj, Sandip Kudesia}

Department of Pathology, SGRRIMHS, Dehradun, Uttarakhand, India

Address for the Correspondence: Dr. Brijesh Thakur, Department of Pathology, SGRRIMHS, Dehradun, Uttarakhand, India E-mail: drbrijeshthakur03@ gmail.com

Access this article online
Website: www.ijmedph.org
DOI: 10.4103/2230-8598.151277
Quick response code:

\section{CASE REPORT}

A 70-year-old man presented in surgical out-patient department with a solitary subcutaneous lump in the right lower extremity since 2 years. The lump had been growing slowly to the present size of approximately $5 \mathrm{~cm} \times 3 \mathrm{~cm}$. There was no history of pain, any type of discharge, prior trauma, or surgical intervention. The patient had no preceding history of weight loss, bone pain, jaundice, abdominal pain, chest pain or cough to suggest a metastatic lesion. There was no contributory family history or similar lesion.

Local examination revealed a soft to firm subcutaneous lump of $5 \mathrm{~cm} \times 2.5 \mathrm{~cm}$ present in the lateral compartment of right leg. It was well defined, mobile and nontender even on deep palpation. Clinically there were no signs of foot drop, vascular or neurological deficit. Plain radiograph revealed a soft tissue mass at the lateral aspect of the right leg with no bone involvement. Hematological investigations showed mild leukocytosis $\left(12,400\right.$ cells $\left./ \mathrm{mm}^{3}\right)$ with mild peripheral eosinophilia $(14 \%)$. Other parameters were within normal limits. There was no evidence of any hemoparasite. Fine-needle aspiration cytology was done which yielded paucicellular hemorrhagic aspirate. Excision of lump was done under local anesthesia and tissue was sent for histopathological examination to the department of pathology. Written informed consent was taken from the patient prior to the surgical procedure.

Grossly, excised globular whitish piece of tissue measuring $5.6 \mathrm{~cm} \times 2.8 \mathrm{~cm} \times 1.8 \mathrm{~cm}$ was received. Cut surface was yellowish white with few small dilated spaces and tiny foci of hemorrhage [Figure 1a]. One whole cross section of tissue was taken, fixed in 10\% formalin and processed. Paraffin-embedded sections showed smooth muscle bundles, collagen bundles, proliferating fibroblasts with variably sized capillaries as well as numerous small to medium sized and few thick-walled blood vessels. Focal areas showed lobules of mature adipose tissue and few hemorrhagic foci [Figure 1b and c]. Histology established the diagnosis of AML. Masson Trichome stained section confirmed smooth muscle proliferation and collagen deposition [Figure 1d]. 

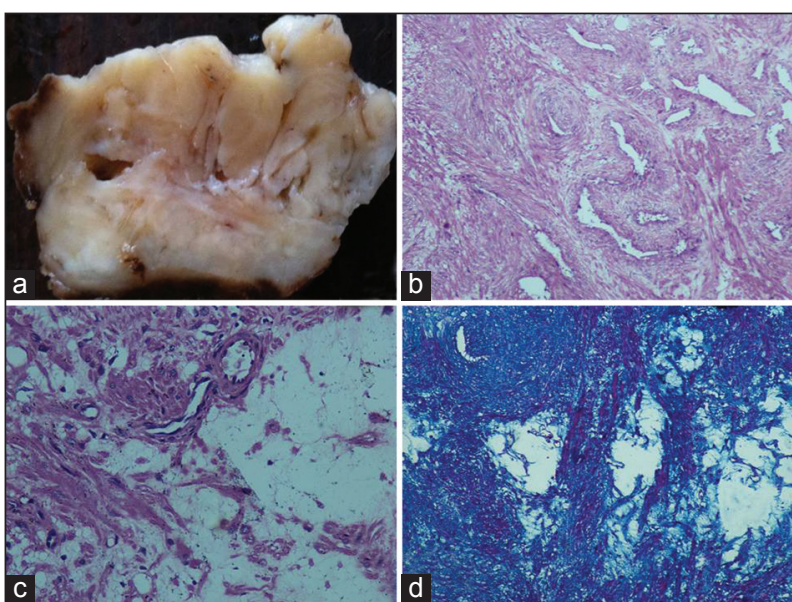

Figure 1: (a) Excised specimen showing yellowish white cut surface with few small dilated spaces and tiny foci of hemorrhage (b) section showing smooth muscle bundles, proliferating fibroblasts with few small to medium sized blood vessels ( $\mathrm{H}$ and $\mathrm{E}, \times 100)$ (c) focal area showing mature adipocytes with few small capillaries $(H$ and $E, \times 400)(d)$ section showing smooth muscle proliferation and collagen deposition (Masson Trichome, $\times 100$ )

\section{DISCUSSION}

Angiomyolipomas have been classified in the perivascular epithelioid cell neoplasms (PEComa family). PEComas are themselves a kind of mesenchymal tumor that involves cells that form the connective tissue, cardiovascular and lymphatic systems and defined by the presence of distinctive perivascular epithelioid cells. ${ }^{[2]}$ Older literature may classify them as hamartomas or choristoma. Some molecular studies have demonstrated its clonality, and immunohistochemical and ultrastructural studies support the idea of histogenesis from a single cell type ${ }^{[1]}$ The etiology and pathogenesis of the neoplasm are unknown.

Three methods for detecting AML are ultrasonography, computed tomography (CT) and magnetic resonance imaging (MRI). Ultrasound is more sensitive for fat content while CT gives fast, detailed and accurate measurement. MRI is safer and avoids radiation exposure. Extrarenal AML preferentially occur in older men and are never associated with tuberous sclerosis. These are small, seldom larger than $40 \mathrm{~mm}$. Grossly, the tumor is yellow to gray depending on the relative proportion of the various tissue elements. They show mature SMC while in renal AML show HMB45-positive epithelioid SMC. Aggregates of lymphocytes are more frequent in extrarenal AML. Hence, we propose to distinguish these soft tissue AML from AML of the kidney and liver.
In the present case, other differential diagnosis was thought to be angioleiomyoma (AL). Indeed, the histologic features of extra-renal AML mimic AL except for the fatty element, and both tumors occur in either dermis or subcutis. Moreover, AL is known to rarely contain a small amount of fat cells. ${ }^{[6]}$ Paroxysmal pain characteristic of AL was not seen in the present case.

Treatment usually consists of marginal excision of the mass. These tumors are rarely diagnosed before surgery because they are not commonly evaluated with cross-sectional imaging, and sometimes imaging findings are not tumor-specific. Many a times, clinically they are considered as lipoma. Occasionally, extensive fibroplasia and the bizarre appearance of many proliferating fibroblasts are considered to be attributed to local host reaction and degenerative changes. However, these changes may necessitate these tumors to be differentiated from a lipo or leiomyosarcoma. ${ }^{[2]}$

To summarize, AML should be considered as a differential diagnosis for a well-demarcated subcutaneous lump even when radiological findings are nonspecific or inconclusive. They should be distinguished from renal AML with respect to clinicopathological and immunohistochemical features. The pathogenesis of extrarenal AML has yet to be answered and the choice of management necessitates a weighing of risks based on the association with recurrence and other clinicopathological features.

\section{REFERENCES}

1. Eble JN, Sauter G, Epstein JI, Sesterhenn IA. World Health Organization Classification of Tumours: Pathology and Genetics of Tumours of the Urinary System and Male Genital Organs. Lyon: IARC Press; 2004.

2. Mahera H, Giamarelou N, Karabela-Bouropoulou V, Samoilis S, Anagnostopoulos D. Soft tissue angiomyolipoma. A case report. Arch Anat Cytol Pathol 1997;45:221-6.

3. Simonato A, Maffezzini M, Lodolo C, Raber M, Carmignani G, Bussani R. Extrarenal angiomyolipoma: Vascular, lymphonodal, and perirenal localization. Report of 4 cases. Arch Ital Urol Androl 1994;66:129-32.

4. Del Sordo R, Colella R, Leite S, Sidoni A. Cutaneous angiomyolipoma: A case report and literature review. Pathologica 2005;97:137-40.

5. Kim Y, Kang Y, Lee ES, Kim A. Angiomyolipoma of the lower lip: A case report and review of the published work. Basic Appl Pathol 2011;4:18-20.

6. Watanabe K, Suzuki T. Mucocutaneous angiomyolipoma. A report of 2 cases arising in the nasal cavity. Arch Pathol Lab Med 1999;123:789-92.

How to cite this article: Thakur $B$, Kishore $S$, Bhardwaj $A$, Kudesia S. Extrarenal angiomyolipoma in an elderly male: A case to remember. Int J Med Public Health 2015;5:121-2. Source of Support: Nil, Conflict of Interest: None declared. 